\title{
Analysis on the Optical Fiber Transmission Maintenance and Management in Communication Engineering
}

\author{
Chuan $\mathrm{He}^{1, \mathrm{a}}$ \\ ${ }^{1}$ ChongQing College of Electronic Engineering, ChongQing, 401331,China \\ ahechuan023@sina.com
}

Keywords: Communication engineering; Optical fiber transmission; Maintenance; Management.

\begin{abstract}
The emergence of the optical fiber communication technology, it is injected fresh blood to this new era. Because it has a long transmission distance, large capacity of communication, the advantages of strong anti-interference ability, in the field of radio, telephone and computer networks and other have very good application, the major benefit of the mankind, showed quite outstanding in the field of communications. With the rapid development of social information, and the popularity of the Internet applications, more and more is also high to the requirement of optical fiber communication transmission, optical transmission in optical fiber communication technology in the practical application shows excellent performance, its application prospect will be broader. This article will analyze the necessity of optical fiber transmission in the communication engineering maintenance, and expounds the principle of optical fiber communication system in optical transmission equipment maintenance, at the same time focus on the analysis of optical transmission equipment maintenance.
\end{abstract}

\section{Introduction}

The optical fiber communication system is mainly composed of optical cable, photoelectric converter, fiber optic repeater, electro-optical converters, and computers. Due to the electrical signal is output in the computer, and the optical signal is on optical fiber transmission, therefore, you need to add the photoelectric conversion device on the computer terminal system, to make the conversion between different signals. Can realize optical signals into electrical signals is a photoelectric converter, and realize the electrical signals into light signals is electro-optical converters, selection for optical fiber simplex communication mode, if in a full-duplex communication between two end systems, you should need two root fiber. Fiber optic repeater can be made to extend the length of the fiber, effectively prevent the attenuation of the signal, so that the farther distance transmission.

\section{The characteristics of optical fiber time delay}

The characteristics of optical fiber communication technology. (1) The very wide frequency band, large capacity of communication. About $50000 \mathrm{GHZ}$ fiber available bandwidth, transmission bandwidth is much larger than copper wire or cable, for all kinds of broadband information transmission is of great significance, otherwise, cannot satisfy the future broadband integrated services digital network (B ISDN) development. For single wavelength optical fiber communication system, due to the electronic bottleneck effect of terminal equipment can't play to the advantages of optical fiber bandwidth is big. Usually used in a variety of complex technology to increase transmission capacity, especially now of DWDM technology has greatly increased the transmission capacity of the fiber. At present, the transfer rate of the single wavelength optical fiber communication system is in commonly 2.5 Gbps $~ 10$ Gbps.

(2) Low dissipation, long distance relay. At present practical quartz optical fiber loss can be less than $0.2 \mathrm{~dB} / \mathrm{km}$, is lower than any other losses in the transmission medium, if in the future use of quartz is extremely low loss optical fiber, the theoretical analysis of loss can be decreased from 10 $9 \mathrm{~dB} / \mathrm{km}$. Due to the low loss optical fiber, so can realize long distance relay, composed of quartz optical fiber optical fiber communication system maximum relay distance can reach more than 200 $\mathrm{km}$, consisting of the quartz is very low loss optical fiber communication system, its maximum 
relay distance can amount to thousands or even tens of thousands of kilometers, this means that through the optical fiber communication system, can cross a larger distance for a long distance transmission lines, due to the reduction in the number of Repeaters, system can greatly reduce the cost and complexity.

(3) The electromagnetic interference resistance ability. Optical fiber raw material is made of quartz insulator material, not easily corroded, and good insulation. Associated with it is an important characteristic of the optical waveguide immunity to electromagnetic interference, it is not affected by the thunder and lightning interference of nature, also is not affected by anthropogenic emissions of electromagnetic interference, it may also be used with the high voltage transmission line parallel erection or and power conductor composite constitute a composite fiber optic cable.

(4) No crosstalk interference, good secrecy. Light waves in optical fiber transmission, it is difficult to leak out from the optical fiber, even in a corner, the bend radius is very small, the light leakage is also very weak, if on the surface of fiber or cable coated with a layer of flatting agent effect is better, in this way, even if the total number of fiber in optical fiber, no crosstalk interference, can also be based on the outside of the fiber optic cable, hacking into the optical fiber transmission of information.

\section{The Necessity of the optical transmission equipment maintenance in the optical fiber communication system}

Both in what kind of scientific instruments and industrial equipment maintenance, after the need to use and maintenance methods become each must consider the question after the equipment research and development, optical transmission equipment is not exceptional also, also needs to be maintained, but the maintenance is methodology, wherefore they roughly means something not bad, don't hurt, will inevitably cause damage removal, visible, the maintenance methods if not when also can cause damage to equipment, can only maintain equipment, correct maintenance methods are debugging, or be called. Keep a good running environment including equipment power supply quality, computer room environment temperature, humidity, dust and so on is in line with the requirements for these equipment life, reduce the failure rate is the important premise in general, modern communication equipment is more demanding on the environment.

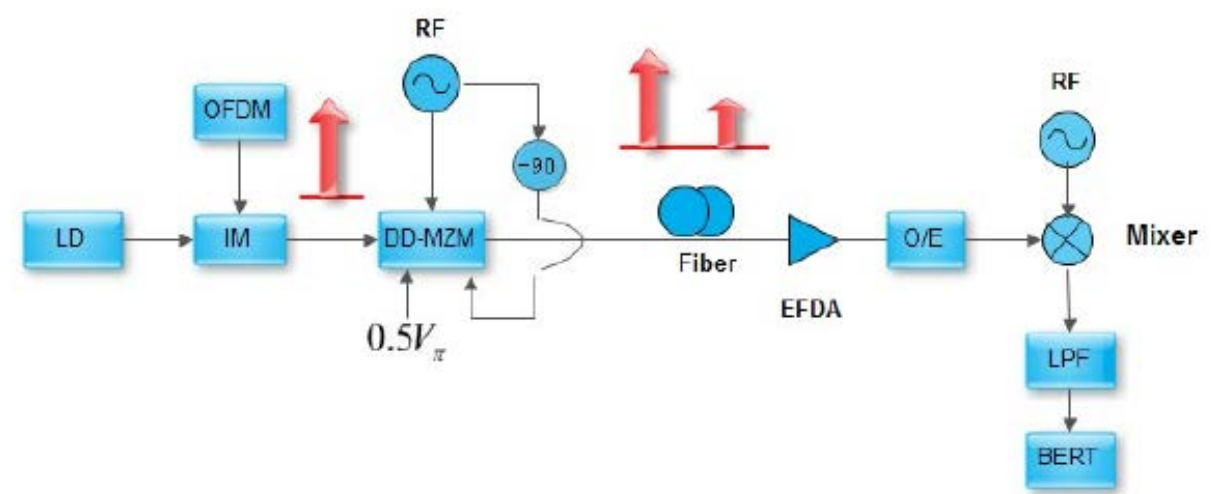

Fig. 1 single side band optical carrier modulation system schematic diagram

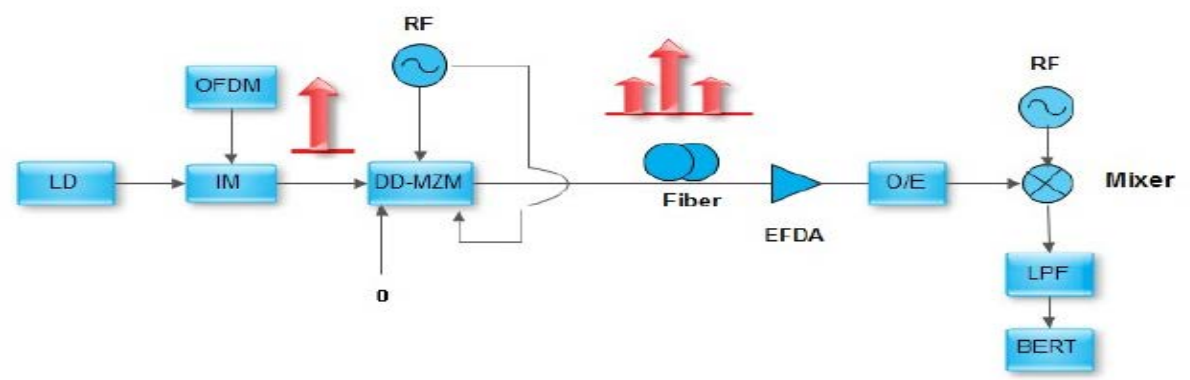

Fig. 2 double sideband modulation system schematic diagram 


\section{The principles of optical transmission equipment maintenance in the optical fiber communication system}

(1) The external first, after the transfer. When fault occurs, we determine the cause of the failure, from the external inspection, such as whether the cable connection is good, if there is a line break, etc., can be based on past experience, first check equipment are prone to failure part, if outside there is no problem, then continue to check the internal transport, such examination can reduce the damage to the equipment, equipment in the future work, prolong service life.

(2) After the first single station, veneer. When trying to identify the occurrence of fault location, first find out fault from the within a site, and then screening was carried out on the site, from which a piece of board, determine the fault screen regularly work step by step, can't blind to see, and have no idea of view will waste the time to repair, thereby causing loss to the equipment.

(3) After the first line, branch. In optical transmission equipment, circuit board failure often causes branch board of the faults. When the fault is location, you need to follow the principle of "first line, after the branch".

(4) The first repair, after repair. In the event of a failure, the system maintenance personnel must first using the alternate routing or idle resources repair business, fault repair as much as possible and then shorten the time delay of fault.

In optical transmission equipment alarm troubleshooting tips, first determine the level of alert, crisis warning suggests fault degree of serious and affect the communication, delay too long is easy to cause big problems. So, follow the principle of advanced first, low-level, first to deal with emergency accidents, general warning screen again after can be finished in a accident and emergency repair. Fault handling must points clear of the emergency and the secondary, ensure higher failure to get timely maintenance, lest affect to expand.

At present our troubleshooting of optical transmission equipment, there are several kinds of commonly used methods: alarm performance analysis method, replacement method, ring back, configuration, data analysis, instrument testing method, these methods are commonly used detection of equipment failure, according to the different types of fault to choose the appropriate solution, ensure the normal operation of optical transmission equipment.

\section{The maintenance way of the optical transmission equipment}

Reduce artificial barriers. In the modern communication equipment maintenance, usually do not use again that trivial test adjustment, such as quarterly test on test and so on, now only need to take preventive surveillance monitoring means, in zero fault status or without apparent failure phenomenon, it is best not to touch the machine equipment, casually can make to reduce artificial barriers.

Keep equipment in good running environment. The merits of the quality of power supply equipment, and computer room environment humidity, temperature, dust, etc have great relevance, is mainly to reduce equipment failure rate, extend the life of the equipment. Generally speaking, the current communication equipment of environmental requirements is more stringent.

The fault plug tray is an important means of processing equipment circuit fault. Have certain cases as spare some easy bad delicate dish/plug-in. For machine set higher integration, conductor is slender, assembly is quite dense, sometimes we cannot fix by oneself, or is likely to lead to the entire machine plate scrapped the occurrence of damage, if I find fault after should be the first time to contact, returned to the factory to repair it.

Should fully exert the effectiveness of network management system actively. In the current communication system, network management functions are perfect, it can be in the business of uninterrupted time for real-time performance indicators to monitor, not only can monitoring the fault, but also to determine the fault type, even can locate fault effectively, in terms of preventive maintenance and troubleshooting is quite remarkable. In new era of communication, software technology plays a very important role, equipment many function is difficult to achieve both 
reliable software into reality, so there was no related technologies mastering it is impossible to control the modern communication technology.

The fault handling of modern optical transmission system. a. The substitution method. Replacing method is a doubt that work is not normal objects by using a work item in for, finally realizes the fault location and the effect of troubleshooting. Is also a kind of relatively common fault handling, replacing method can be used in the transmission of external equipment problems ruled out, such as optical fiber, switches, power supplies, relay cable, etc., or a single fault location in the station, veneer of single station is ruled out, a failure problems, such as a piece of plate can work to take the normal similar plate for replacement.

b. The observation analysis. When there was a fault in the system, will show the corresponding to each other in network management and equipment of warning signs, warning lights on through to the equipment running status, can immediately find the malfunction, and network management will be record to the alarm event information and performance data, through the analysis of the information, combined with the principle of alarm mechanism in the SDH frame structure with overhead bytes, can preliminarily concluded that the fault type and fault point.

c. The instrument testing method. Usually used in the transmission equipment outside of the problems and issues with other equipment docking effectively ruled out. Especially the light path fails, take the optical power meter test plate glow with lines of light, can quickly judge the plate or line problem, OTDR cable fault point can be selected quickly orientate accurately.

\section{Summary}

In a word, in the unceasing progress distance, facing problems, and actively and solve the problem, combining past experience and science and technology, promote the optical fiber communication equipment constantly improve, rapid development of electric power communication field. With the progress of science and technology, the operation of the optical fiber communication network, is to rely on network management and advanced science and technology surveillance system, also must pay attention to the quality of equipment management, attaches great importance to the protection work, provided a basis for fiber optic equipment in good running, little of contribution to the progress of social power.

\section{References}

[1] Mallat S,Huang WL.Singularity detection and processing with wavelets. IEEE Transactions on Information Theory. 2012(10).

[2] Zarowski C J.Connection Splice Fault Location in Fibre Optic Cables. Canadian Conference on Electrical and Computer Engineering. 2011(10).

[3] Fenglei Liu,,Zarowski C J.Events in Fiber Optics Given Noisy OTDR-Data. I:GSR/MDL method. IEEE Transactions on Instrumentation and Measurement. 2012(08).

[4] S.M.EISAFTY,M.A.SHARKAS.Identification of Transmission Line Faults Using Wavelet Anal- ysis. IEEE Circuits and Systems Magazine. 2013(01).

[5] E.SAMI,Y.SELCUK,P.MUSTAFA.Energy and Entropy-based Feature Extraction for Locating Fault on Transmission Lines by Using Neural Network and Wavelet Packer Decomposition. Expert Systems with Applications. 2013(10).

[6] R.G.Redinbo,Cung Nguye.Concurrent Error Detection in Wavelet Liftingt Ransforms. IEEE Transactions on Computers. 2014(02). 